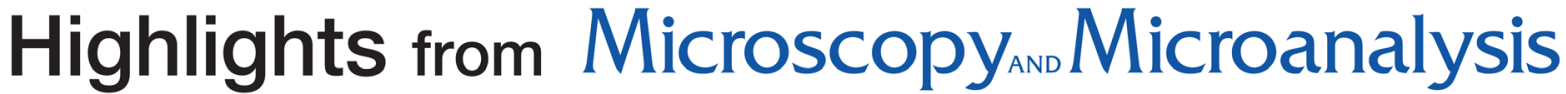

\section{Techniques Development}

Plasma focused ion beam serial sectioning as a technique to characterize nonmetallic inclusions in superelastic Nitinol fine wires by JL Gbur, R Kelley, and JJ Lewandowski, Microsc Microanal | doi:10.1017/S1431927620024617

Understanding the fatigue behavior of superelastic (SE) Nitinol fine wires $(<140 \mu \mathrm{m}$ diameter) requires the ability to accurately characterize the connectivity and distribution of nonmetallic inclusion (NMI) populations within the wire volume. Traditional two-dimensional (2D) metallographic techniques lack the resolution necessary to identify NMIs at micron and submicron sizes. Furthermore, 2D techniques alone will not provide accurate NMI connectivity or feature quantification because analysis relies on the sectioned location. This work investigated the application of plasma focused ion beam (PFIB) serial sectioning with scanning electron microscopy (SEM) in high purity (HP)-lower oxygen content and standard purity (SP)-higher oxygen content SE Nitinol wires. Segmented, three-dimensional (3D) visualizations clarified NMI and pore connectivity as well as provided quantification of the area, volume, and distribution of NMI populations (Figure). Published work on the fatigue of SE Nitinol wires reveals NMIs as sites of fatigue crack initiation. Data collected from this work are being used to rationalize the effects of purity on the fatigue lifetime of these materials.

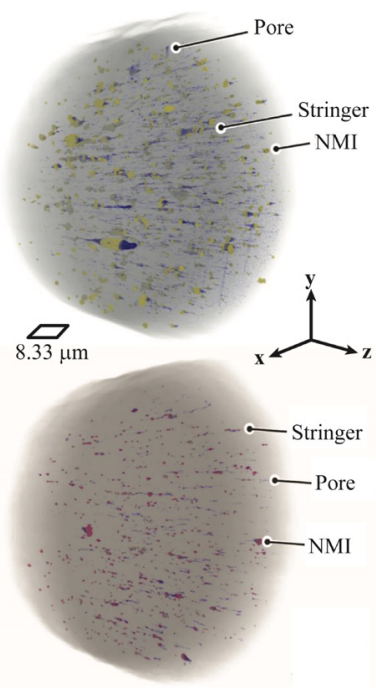

Segmented 3D visualization of SP Nitinol (top) and HP Nitinol (bottom) showing the distribution of NMIs, pores, and stringers in the wire volume.

\section{Material Applications}

Inelastic Scattering in Electron Backscatter Diffraction and Electron Channeling Contrast Imaging by BG Mendis, J Barthel, SD Findlay, and LJ Allen, Microsc Microanal | doi:10.1017/S1431927620024605

Electron backscatter diffraction (EBSD) and electron channeling contrast imaging (ECCI) are two scanning electron microscopy-based techniques that provide crystallographic information, such as sample orientation, strain, and defects (for example, dislocations and stacking faults). The techniques use the dependence of the backscattered electron signal on "channeling," the phenomenon where the incident electron beam is attracted to the positive potential of the atom columns as it propagates through the specimen. The useful EBSD and ECCI signal is superimposed on a large background, which carries no sample-related crystallographic information. The background is due to backscattered electrons that have lost large amounts of energy to the sample. In this paper, we simulate the role of small energy loss events, such as plasmons, on the EBSD and ECCI signals (Figure). It is shown that the EBSD contrast is maximum after a few plasmon scattering events, while ECCI is largely unaffected. These results can be explained by considering the effect of the plasmon scattering angle on the intrinsic contrast mechanisms in both EBSD and ECCI.

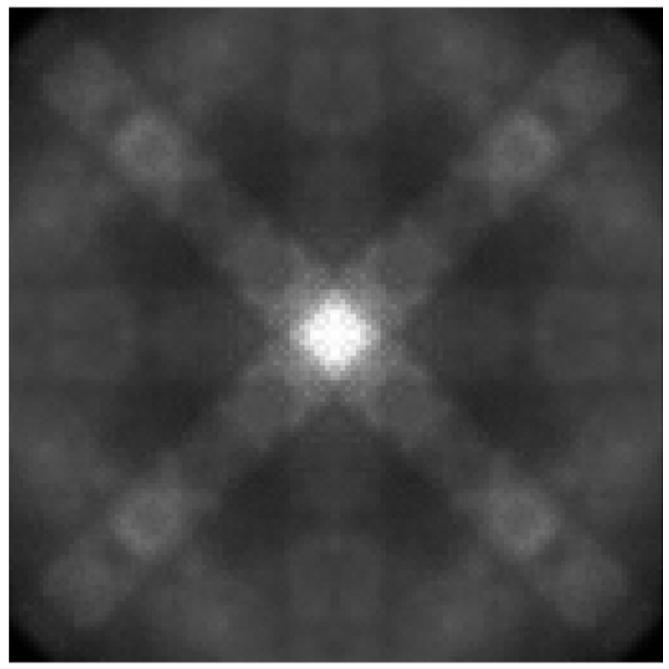

[001]-silicon EBSD pattern simulated using a multislice algorithm that includes phonon and plasmon excitations. The contrast of the Kikuchi bands, generated by phonon scattering, first increases with plasmon excitation before decreasing for multiple plasmon scattering events. 


\section{Microscopy Microanalysis}

\section{Biological Applications}

FE-SEM characterization of $\alpha$-mannose density and surface mapping changes in human sperm head during in vitro capacitation by MJ Gómez-Torres, L Robles-Gómez, N Huerta-Retamal, P Sáez-Espinosa, M Avilés, J Aizpurúa, and A Romero, Microsc Microanal | doi:10.1017/S1431927620024630

Glycocomponents develop significant molecular activity in human gamete transport, recognition, and interaction. The redistribution of $\alpha$-mannose in sperm membranes is an example of the changes that occur during sperm capacitation. However, it is still unclear how in vitro capacitation affects the $\alpha$-mannose residues and their spatial distribution in the sperm glycocalyx. We characterized $\alpha$-mannose density, and specific acrosomal and postacrosomal locations, before and after in vitro capacitation (one to four hours) by means of high-resolution field emission scanning electron microscopy (FE-SEM) and Concanavalin A (Con A) lectin gold labeling (10 $\mathrm{nm}$ diameter). Our results showed that $\alpha$-mannose residues were present preferably on acrosome domains before and after in vitro capacitation. Nevertheless, we noted a progressive and time-dependent decrease of $\alpha$-mannose during in vitro capacitation (Figure). Topographical mapping also allowed identification of an unexpected relocation toward acrosomal domain apical areas after capacitation. The novel combined approach offered an accurate method to locate $\alpha$-mannose residues and new details of human sperm biomolecular and structural dynamics during capacitation events.
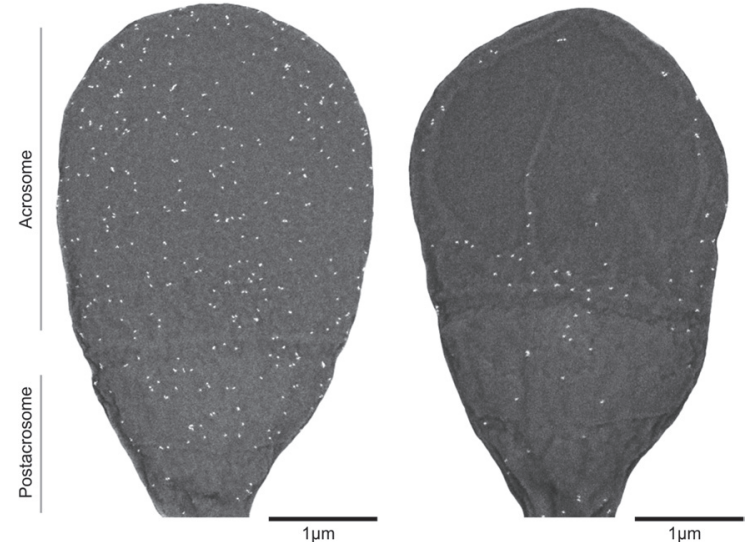

FE-SEM micrographs of incapacitated (left) and four-hour-capacitated (right) human sperm. Acrosome (indicated by the top line on left of figure) and postacrosome (bottom line) domains are shown. Note the decrease in gold nanoparticle density after four hours of in vitro capacitation (right) and the gold nanoparticle redistribution to the acrosomal apical area.

\section{A top journal in Microscopy}

\section{Published for the Microscopy Society of America}

Editor: John Mansfield, University of Michigan, USA

The only journal owned by scientists and published for scientists, Microscopy and Microanalysis provides original research papers in the fields of microscopy, imaging and compositional analysis. This distinguished international forum is intended for microscopists in both biology and materials science.

\section{Online submission at cambridge.org/mam/submit}

View the journal online at cambridge.org/mam

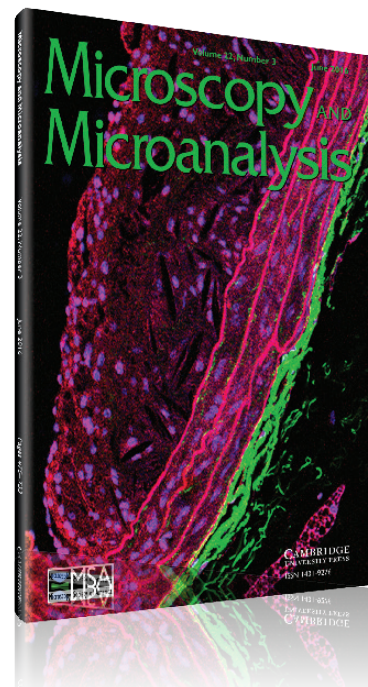

CAMBRIDGE UNIVERSITY PRESS 